\author{
Gdańsk 2018, Nr. 39 \\ https://doi.org/10.26881/sgg.2018.39.05 \\ Werner Abraham \\ Wien-München
}

\title{
Valenzdiversifikationen: Was ist Thetikvalenz?
}

Thetische Sätze inkorporieren zum Unterschied von kategorischen Sätzen alle Argumente in VP, sehen also kein Vorfeld für anaphorisch-thematische DPs und insbesondere Subjekte über der ersten Verbklammer (Comp) vor. Es wird den Vorstellungen von Kuroda nachgegangen, der meint, dass Thetik und Kategorik im Japanischen direkt und unmissverständlich durch die Kasussuffixe - ga bzw. -wa angezeigt werden. Nach solchen Vorstellungen zeigt das Deutsche, das ja diskursgebundene Sätze nicht direkt morphologisch von diskursungebundenen Sätzen unterscheidet, keine direkten Entsprechungen zur ga-wa-Syntax und entsprechend nicht zur Thetik-Kategorialitätsunterscheidung. Gehen wir aber davon aus, dass reine VP-Sätze im Deutschen ein Verständnis von Konstruktionsvalenz abbilden, d.h. wenn sich von Thetikvalenz (Nullvalenz bei voller Satzsyntax) gegen kanonische Kategorikvalenz sprechen lässt, dann geraten andere Satzbegriffe in seinen Bannkreis einer solchen Konstruktionsvalenz. Diesen Konstruktionstypen geht der folgende Artikel nach.

Schlüsselwörter: thetisch, kategorisch, Suvbjekt-VP-Inkorporation, Konstruktionsvalenz, Saztzdiskursbindung, japanisch $-g a /-w a$

Valence disversifications: what is thetic valence? - Unlike categoriacal sentences, thetic sentences incorporate all arguments in VP. As a consequence, thetics have no anaphoric-topicalized DPs and in particular canonical subjects to the left of the first verbal bracket (Comp). We pursue ideas developed by Kuroda, who believes that thetics and categoricals occur directly and unambiguously in Japanese marked by the case suffixes - ga and -wa. According to these ideas, German cannot equivalently, i.e. directly mark discourse bound sentences as different from discourse unbound sentences. Thetics and categoricals are totally alike in German with respect to their discursive status. However, given that VP-incorporated arguments map zero valence and nevertheless are fully satisfied constructions, we can speak of construction valence. We discuss sentence types that comply with VP-internal arguments and without a deep structure of discursive status.

Keywords: thetic, categorial, subject-VP-incorporation, constructions valency, discourse status of sentence, Japanese -ga/-wa

\section{Vorverständnis und implizite Widmung}

Die Zuweisung von Valenzkasus geschieht grammatiktheoretisch durch DP-Lizensierung. Die Bedingung für eine solche Lizenz nennt man syntaktische Kasusbedingung. Sie besteht, dies ist ebenso verständlich, eine Beziehung zu morphologischer Kasusmarkierung. Dazu gibt es drei Möglichkeiten (vgl. etwa McFadden 2004, Koenig/Michelson 2015): 
(i) Kasusmorphologie ist die direkte Ausbuchstabierung syntaktischer Kasusmerkmale.

(ii) Kasusmorphologie bezieht sich auf syntaktische Kasusmerkmale, kann aber auch durch andere Faktoren mitbestimmt sein.

(iii) Kasusmorphologie ist ohne Bezug auf syntaktischen Kasus bestimmt.

Ich nehme aus der Literatur drei methodische Valenzstandpunkte auf, die mir wichtig erscheinen und die ich unter Beachtung der drei Kriterien (i)-(iii) weiterverfolgen will. Alle haben mit jener Valenzbegrifflichkeit zu tun, die Engel $(1996,2004)$ nach Tesnière (1959, 1980) zusammen mit anderen weiterentwickelt hat. ${ }^{1}$ [„Ergänzungen“ werden in der Folge „Objekte“ genannt.]

1.1. „Wenn es Ergänzungen gibt, die bestimmten Elementen subklassenspezifisch zugeordnet werden, dann müssen diese Elemente in geeigneter Weise markiert sein, sie müssen eine Eigenschaft aufweisen, die sie als geeignet für die Kombination mit den Ergänzungen ausweist; eine Eigenschaft, die spezielle Ergänzungen verlangt oder wenigstens zulässt und damit andere Ergänzungen ausschließt. Diese Eigenschaft nennen wir Valenz" (Engel 1996: 35). - Hauptpunkt: individuelle Beziehung zwischen Verb und Ergänzung, wobei offenbleibt, ob diese Beziehung semantischer oder flexionsmorphologischer Natur ist bzw. sein muss.

1.2. Wenn Zeichen des Typs $\mathrm{X}$ regelmäßig zusammen mit Zeichen des Typs $\mathrm{Y}$ auftauchen, kann dies auf zweierlei Art erklärt werden: (i) X ist anhand der Wertigkeit von Y erforderlich oder (ii) Es liegt eine komplexe Konstruktion $\mathrm{Z}$ vor, die $\mathrm{X}$ und $\mathrm{Y}$ erfordert. Ich argumentiere, dass bei der Entscheidung zwischen (i) und (ii) entschieden werden sollte, ob die Durchschnittsmengen von $X$ und $Y$ prototypischen Instanzen von (i) ähneln. Der Gedanke dahinter ist, dass die Eigenschaften des Prototyps (i) zeigen, dass die Wertigkeit Wortformen (wie Plural) morphosyntaktisch nachzeichnet. Ähneln also die Durchschnittsmengen von X und Y nicht dem Prototyp (i), dann würden sich falsche morphosyntaktische Parallelen ergeben. Und andererseits würden sich Lücken in der Parallelität ergeben, wenn das Phänomen als Instanz (ii) identifiziert würde. ${ }^{2}$ Hier geht es also um konstruktionell Gemeinsames und Unterschiedliches bei der morphologischen Verbklassifikation. - Hauptpunkt: konstruktionelle Umgebungs(un)abhängigkeit der Prädikat-Argument-Beziehung.

1 Zu den wissenschaftlichen Veröffentlichungen aus der Hand des Jubilars vgl. Kątny, Andrzej / Schatte, Christoph (Hg. 1999): Das Deutsche von innen und von außen. Poznań. Sowie Eichinger, L. / Kubczak, J. / Berens J. (Hg. 2011): Dependenz, Valenz und mehr. Tübingen. - Ich danke einem Gutachter für wertvolle Hinweise.

2 Das englische Original lautet: „If signs of type $\mathrm{X}$ regularily cooccur with signs of type $\mathrm{Y}$, this can be explained in two ways: (i) $\mathrm{X}$ is required by the valency of $\mathrm{Y}$, or (ii) there is a complex construction $\mathrm{Z}$ that requires both $\mathrm{X}$ and $\mathrm{Y}$. I argue that, when deciding between (i) and (ii), grammarians should consider, among other things, whether the cooccurrence of $\mathrm{X}$ and $\mathrm{Y}$ resembles prototypical instances of (i). This recommendation is based on the fact that the properties of the prototype of (i) show that valency is a morphosyntactic feature of word forms (like plural). Therefore, if the cooccurrence of $\mathrm{X}$ and $\mathrm{Y}$ does not resemble the prototype of (i), treating it as an instance of (i) would establish a wrong parallel to morphosyntactic phenomena. And, on the other hand, if it does resemble this prototype, treating it as an instance of (ii) would miss this parallel. " (Jacobs 2009 Abstract) 
1.3. Es erscheint durchaus üblich, dass in vielen Sprachen mit offener Kasusmarkierung einige direkte Objekte kasusmarkiert erscheinen, andere jedoch nicht, und zwar je nach den semantischen und pragmatischen Funktionen des Objekts. Nach Bossong (1991) nennt man dieses Phänomen 'Differential Case Marking/DOM', deutsch "morphologische Kasusdifferenzierung / MKD". ${ }^{3}$ Dabei handelt es sich klar um paradigmatische Valenz, also Kasusvariation in Abhängigkeit nicht von der syntaktischen Satzposition oder -funktion, sondern von anderen Kriterien wie Belebtheit bzw. der Belebtheitsskala ,Human > Animate > Inanimate (wie im Singhalesischen) und referentielle Definitheit bzw. der Definitheitsskala ,Personalpronomen $>$ Eigenname $>$ definite NP > indefinite spezifische NP > nichtspezifische NP (Hebräisch; nach Aissen 2000). Auch das Personskriterium nach der Skala ,(Singular und Plural) 1. „ich“ / 2. „du“ > 3."er-sie-es “" spielt eine Rolle. Diese Skalen signalisieren das Phänomen, dass Objektreferenz mit höherer (links-vor-rechts) Markierung eher entsprechend kasusmarkiert erscheint. Kriterienqualität und deren Verteilung ist für über 300 Sprachen belegt (Bossong 1985). - Hauptpunkt: Ein und dieselbe Satzgliedposition wird nach zwei oder mehreren (semantischen oder referentiellen) Kriterien anhand verschiedener Kasusmorphologien markiert.

Alle in der Folge diskutierten Erscheinungen umspielen diese drei Auffassungen von Valenz (Wertigkeit), dies mit folgenden Zielen:

(i) Verstehen wir Valenz, also als paradigmatische oder als syntagmatische Erscheinung?

(ii) Beziehen wir zur Valenzbegrifflichkeit thetische Sätze mit ein?

(iii) Was für eine Rolle spielt Valenz in Sprachen, in denen diskursgram-matische Argumentthematisierung deren Grammatik beherrscht?

Wir orientieren uns in der Folge an diesen drei Fragen.

\section{Objektvalenz und Diathese}

\subsection{Paradigmatische gegen syntagmatische Valenz: Diathesenvalenz}

Valenz lässt sich paradigmatisch und syntagmatisch verstehen (Abraham / Leiss 2012, 2017; Abraham 2016). Diese Unterscheidung ist aber in der Valenzliteratur so gut wie unbeachtet geblieben - wenn wir von Bossongs (1991) und Aissens (2000) „DOM“ absehen, die aber diesen grundlegenden theoretischen Unterschied trotz gleicher Phänomenbehandlung nicht erkannt haben. Ich halte dies für die fundamentalste Unterscheidung. Unter paradigmatischer Einordnung versteht sich valenzabhängige Kasusmorphologie als Bedeutungsvarianz über ein und demselben Satzglied, d.h. unter identischer syntaktischer Position. Unter syntagmatischer Einordnung ändert sich die syntaktische Position. Prototypische Beispiele sind

\footnotetext{
3 Im Original: „It is common for languages with overt case marking of direct objects to mark some objects, but not others, depending on semantic and pragmatic features of the object. Following Bossong (1991), I call this phenomenon 'Differential Case Marking/DOM“. (Aissen 2000: 2)
} 
das paradigmatisch kasusvalenzkodierende Russische mit dem Akkusativ gegen den Genitiv für das transitive Objekt, wo der transitive Genitiv Imperfektivität beim Prädikatsverb anzeigt, der Akkusativ hingegen die perfektive Diathesenlesart (Leiss 1991, zum Althochdeutschen Abraham 1997). In (1a-b) sind die Zusammenhänge zwischen morphologischem Kasus und Verbaldiathese einerseits und Nominalreferenz (im Russischen eben nicht durch Artikel signalisiert) andererseits angezeigt (nach Birkenmaier 1977, Brunnhuber 1983; Leiss 1991, 2002a, b; zum Polnischen vgl. Piskorz 2017). In (1) geht es um morphologisch gekennzeichnete Verblexeme (Perfektivpräfix), in (2) um ein und dasselbe Verb, das den Diathesen- und Referenzwechsel per Objektkasus anzeigt. Zu beachten ist jedenfalls, dass der strukturelle Akkusativ (Grundlage zu Passivierung) Affinität zu perfektiven Aspekt und nominaler Individualreferenz herstellt, während der nichtstrukturelle Genitiv zu aspektueller Imperfektivität und indefiniter Nominalreferenz anbindet.

\section{RUSSISCH}

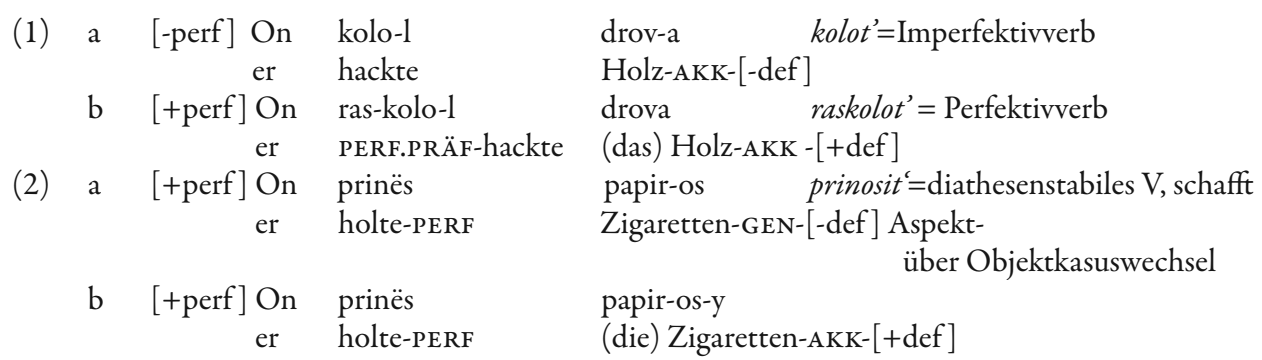

Das Russische setzt wie andere slawische Sprachen keine lexikalisch bestimmte Artikelkategorie. Dennoch sind NPs deutlich in Bezug auf Referentialität interpretierbar, unter anderem durch Diathesemechanismen wie dargestellt in $(1 \mathrm{a}, \mathrm{b})$. Das heißt, der referenzielle Status der Objekt-NPs lässt sich auf der aspektuellen Verbmorphologie eindeutig identifizieren. Imperfektive Verben, wie kolot' in (1a), bezeichnen Nichtreferentialität oder Unbestimmtheit der direkte Objekt- NP im Akkusativ. Akkusativ markiert jedoch spezifische Referenzialität oder Bestimmtheit des NP-Objekts, wenn es von einem perfektiven Verb (raskolot') regiert wird. Es besteht also aspektuelle Affinität zu nominaler Referenz und Kasusmorphologie (Leiss 2002a,b) ${ }^{4}$. Grosso modo (mit den Einschränkungen nach Fn. 4) ist verbale Genitivrektion unvereinbar mit verbaler Imperfektivität.

4 Nach einer Beobachtung des Gutachters sollte eingeschränkt werden, welche Aktionsart vorliegt, die bei Satzperfektivität (bei perfektivem „Viewpont-aspect“) realisiert wird. Beim Paar kolol' - raskolol, wo finitive Aktionsart, gilt das, aber bei der kumulativen AA (Anhäufen einer bestimmten Menge bzw. Masse als Resultat einer Handlung), die ebenfalls zum perfektiven Aspekt gehört, ist der Genitiv in den meisten Fällen die einzige Möglichkeit, während der Akkusativ oft ausgeschlossen ist, vgl.

(i) On nakolol drov Er PERF.PRÄF-hackte Holz-GEN-[+def]

„Er hat (eine bestimmte, ausreichende Menge von) Holz gehackt“ (nur bei Objektgenitiv, jedoch nicht bei Akkusativ. 
POLNISCH:

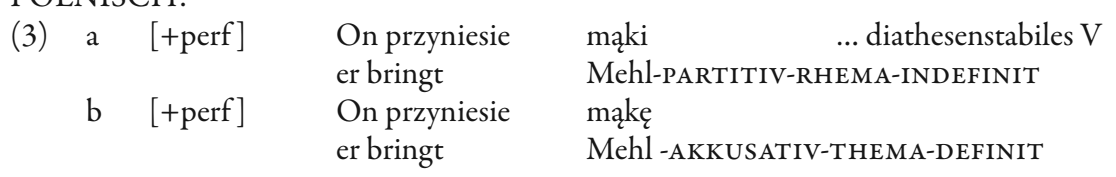

Fehlt jedoch verbale Diathesenmorphologie wie bei polnisch prinosit, dann wird nominale Referenz durch Kasusalternation festgelegt: Objektakkusativ für nominale Definitheit (und Diskursthema), der partitive Genitiv für Indefinitheit und Diskursrhema. In der Affinitätsreihe zwischen Aspekt, Referenz und Kasus zeichnet sich also Asymmetrie ab. Der Genitiv signalisiert Nichtreferentialität nur dann, wenn er von einem Perfektiverb regiert wird. In der Rektion eines Imperfektivverbs bricht Referenz völlig weg. Die Konstruktion wird ungrammatisch (vgl. Piskorz 2017). Dies erinnert auch an die im Finnischen verzeichnete Affinität zwischen dem Partitivkasus und nominaler referenzieller Unbestimmtheit (und Diskursrhematik; vgl. Karlsson 1982). In den Grammatiken identifiziert man auch Resultativität vs. Irresultativität als entscheidende erklärende Diathesenopposition, wie angezeigt in (4)-(5) (nach Karlsson 1982: 101).

\section{FINNISCH:}

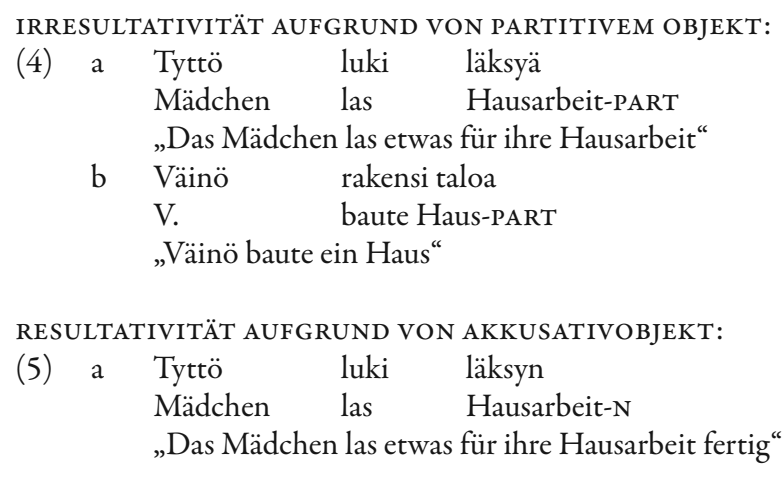

Die Diathesenvalenz des Russischen, Polnischen und Finnischen lässt sich anhand des Deutschen nachvollziehen. Aspektunterschiede waren zwar bereits im Frühmittelhochdeutschen weitgehend eingeebnet. Dafür galt jedoch noch im Spätmittelhochdeutschen der Genitivausdruck für indefinite Referenz so wie im Gotischen dargestellt in (7).

(6) a weil ich $\left[_{\mathrm{VP}}\left[{ }_{\mathrm{DP}}\right.\right.$ das/ein $\left[_{\mathrm{NP}}\right.$ Glas $]\left[_{\mathrm{pp}}\right.$ (des) Wasser(s) $]$ trinke $\left.]\right]$

b weil ich $\left[_{\mathrm{VP}}^{\mathrm{VP}}\left[{ }_{\mathrm{DP}}\right.\right.$ das/ein $\left[_{\mathrm{NP}}^{\mathrm{NP}}\right.$ Glas $]\left[_{\mathrm{PP}}^{\mathrm{PP}}\right.$ (vom) Wasser $]$ trinke $\left.]\right]$

(7) Gotisch (nach Leiss 2000: 150)
[...] jabai hvas matlith
this hlaibis, libaith in ajukduth (J. 6,51; Streitberg 1971)
wer etwas isst
dieses Brotes lebt in Ewigkeit
,Wer etwas von diesem Brot isst, wird in Ewigkeit leben ${ }^{\circ}$

Ähnlich: nanosil vody (brachte ausreichend Wasser - wörtl. Wassers), nabral jagod (sammelte ausreichend BeerenGEN) etc. 
Um den partitiven Genitiv (( ${ }^{*}$ eines/'vom) Wasser $(s)$ trinken $)$ vom referentiell unbestimmten Akkusativ (einen Helden sehen) zu unterscheiden, legt man die folgenden Regeln über das Kriterium [ \pm individuiert] zugrunde. Definitheit wird darüber hinaus mittels des Kriteriums $[ \pm$ spezifisch] identifiziert. Je nach Kontext lässt sich auch nur nach Eigenschaften identifizieren wie in (8d) (Kripkedeskriptor). [ $\mathrm{gdw}=$ genau dann, wenn]

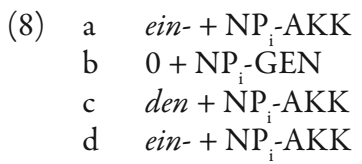
gdw $\mathrm{i}=$ individuiert
... ${ }^{*}$ (einen $)$ Helden sehen
gdw $\mathrm{i}=$ nichtindividuiert
... (*ein) Wasser trinken
gdw $\mathrm{i}=$ individuiert + spezifisch
... *(den) Helden sehen $)$
$\mathrm{gdw} \mathrm{i}=$ spezifisch
... * (einen) Heldenhaften sehen

Daraus ergibt sich eine deutliche asymmetrische Verteilung zwischen Genitivmarkierung und der aspektuellen Eigenschaft des regierenden Verbs. Es besteht Ungleichgewicht auf Kosten der verbalen Imperfektivklasse (indem das Genitivargument der Objektfunktion entzogen wird) und auf Kosten referentieller Unbestimmtheit (die sich fürs Russische nicht über den Objektgenitiv beim imperfektiven Verbbezug ausdrückt). Das Strukturdiagramm in (9) weist die die selektiven Eigenschaften in diesem Affinitätsbezug fürs Russische aus.

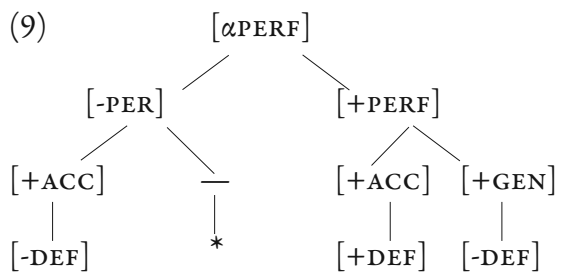

Dieser Mechanismus, mit dem Ausdruck Referentialität der Objekt-NP auf der Grundlage von verbaler Rektionsmorphologie und von Aspekt ist - wie gezeigt - nicht einzigartig fürs Russische. Es gilt auch für andere artikellose Sprachen wie das Tschechische und Serbokroatische, allerdings ohne die gleiche strenge Aspektkomplementarität wie im Russischen. Siehe neben Russisch (1)-(2), die polnischen Beispiele in (3). Auffällig ist, dass das mit Genitiv markierte Argument immer verbadjazent bleibt - nicht also, wie andere kasusmarkierte Argumente unter diskursorientierten Kriterien, vom Verb abgesetzt erscheinen kann.

(10) Diachronie des Artikelausbaus (vaA = verbadjazentes Argument)

\begin{tabular}{|l|c|c|c|c|}
\hline & $\begin{array}{c}1 \text { definiter } \\
\text { Artikel }\end{array}$ & $\begin{array}{c}2 \text { indefiniter } \\
\text { Artikel }\end{array}$ & 3 Genitiv - vaA & $\begin{array}{c}\text { 4 Aspekt- } \\
\text { paradigma }\end{array}$ \\
\hline GOTISCH & - & - & + & + \\
\hline AHD & $+/-$ & $(+)-$ & + & + \\
\hline MHD & + & $+(-)$ & +++ & $+/-$ \\
\hline$($ FRÜH) NHD & + & + & - & - \\
\hline
\end{tabular}


Wie (10) zeigt, hat das Althochdeutsche/AHD noch unsystematisch bestimmte Artikelrealisierungen (nämlich „+/-“ (10), 2. Spalte), während der unbestimmte Artikel fast nicht vorhanden ist („(+)-“, 3. Spalte). Das Mittelhochdeutsche/MHG andererseits zeigt Fortschritt zur Realisierung des bestimmten Artikels (,+ , 1. Spalte) sowie des unbestimmten Artikels („+ (-), 2. Spalte). Zu guter Letzt wird die „Explosion“ der verbalen Genitive („+++“, 3. Spalte) begleitet durch ein diffuses Bild der aspektuellen Morphologie („+-“, 4. Spalte). Die deutsche Gegenwartssprache hat beide Artikelformen, praktisch keine verbale Genitivvalenz noch grammatischen Aspekt. Tabelle (40) zeigt diese relative Verteilung des bestimmten und unbestimmten Artikels. Letzteres ist nicht eindeutig zum unbestimmten im nicht-numerischen und nichtreferenziellen Sinn bereits im Frühneuhochdeutsch fertig geworden. Der bestimmte Artikel ist aus der Demonstrativverwendung entstanden, während sich der unbestimmte Artikel aus dem Zahlwort ein(s) entwickelte.

Was verbaler Aspekt (Diathese), Valenzkasus und nominale Referenz nach der Evidenz aus (1)-(9) gemeinsam haben und was sie dennoch unterscheidet, lässt sich nach mereologischen Kriterien nachvollziehen. Vgl. dazu Leiss (2007a,b), Abraham (2011).

Hauptpunkt dieses Abschnitts: Es ist klar, dass paradigmatisch motivierte Verbrektionsmorphologie eine ganz entscheidende Rolle in vielen Sprachen spielt und sogar im Deutschen gespielt hat. Es ist mit Blick darauf nicht ganz verständlich, wieso dieser Faktor in den deutschen Grammatiken nicht in dieser Spezifik erkannt wurde.

\subsection{Syntagmatisch kodierte Valenz - und übers Deutsche hinaus}

In syntagmatisch kasusvalenzkodierenden Sprachen dagegen gibt es den Zusammenfall des Genitivs und des Akkusativs unter Verbtransitivität nicht: Ein Genitivobjekt ist z.B. nur beim Verb bezichtigen dann möglich, wenn gleichzeitig der transitive Akkusativ (wie in den folgenden Beispielen ihn bzw. sich) gesetzt wird.

(11) a Sie bezichtigten $*\left(\mathrm{ihn}_{\mathrm{AKK}}\right)$ der Untat $_{\mathrm{GEN}}$.

b Er erinnerte ${ }^{*}\left(\right.$ sich $\left._{\mathrm{AKK}}\right)$ seiner Ermahnung $\mathrm{GEN}$.

c Vettel rühmte ${ }^{*}\left(\operatorname{sich}_{\mathrm{AKK}}\right)$, [dass er noch mit kaputten Reifen ins Ziel $\mathrm{kam}]_{\mathrm{GEN}}$.

Hier liegt paradigmatisch kodierte Valenz vor: Kasuswechsel bezieht sich auf eine einzige Satzgliedposition, das transitive Objekt. Der Wechsel über dieser individuellen, einzigen Satzgliedposition hat Diathesefolgen, nämlich Genitiv mit Imperfektivität, Akkusativ mit Perfektivität. Der Wechsel ist die Regel, sofern der Diatheseunterschied nicht bereits durch die Verbform selbst angegeben ist (Perfektivität meist in Form eines Präfixes).

Generell wird das Genitivobjekt im Deutschen nur von wenigen Verben verlangt. Einige dieser Verben sind: sich erinnern, bedürfen, beschuldigen, sich annehmen, sich bemächtigen, gedenken, sich rühmen, sich entledigen. Die Syntagmatik dieser Valenzkasus ist schon dadurch gekennzeichnet, als die Akkusativtivobjekte elliptisch nie fehlen dürfen, will das entsprechende 
Verb als Prädikat eingesetzt werden. Der Genitiv dagegen ist verzichtbar, sofern der direkte Kontext zur Vollinterpretation des Satzes beiträgt. Die indizierten Klammern in $(11 \mathrm{a}-\mathrm{c})$ signalisieren dies. Anders als in den diathesewechselnden Beispielen kodiert das Russische den Genitiv in den (12a-b) nachgedachten Beispielen als PP (präpositionale Gruppe) in den ersten zwei Beispielen zu deutsch (12a-b):

\begin{tabular}{rllll} 
(12) a & $\begin{array}{l}\text { obvinjali } \\
\text { (sie) bezichtigten }\end{array}$ & $\begin{array}{l}\text { jego }_{\text {Akk }} \\
\text { ihn }\end{array}$ & \multicolumn{1}{c}{$\begin{array}{c}\text { v cem-libo } \\
\text { P etwas }\end{array}$} & (PP mit Präpositiv) \\
b & $\begin{array}{l}\text { napomnil } \\
\text { (er) erinnerte }\end{array}$ & $\begin{array}{l}\text { o svoich nastavlenijach } \\
\text { P seiner Ermahnungen }\end{array}$ & (PP mit Präpositiv)
\end{tabular}

und als Instrumentalnomen im Beispiel (13) übertragen aus (11c):

(13) chvalitsja cem (Nebensatz als Instrumental). „sich mit etwas rühmen“

Hier liegt also wie im Deutschen und Russischen eindeutig syntagmatisch kodierte Valenz vor. Das Polnische verwendet an Stelle der Genitivobjekte oft Präpositionalkasus wie in (13a) und (14), regiert durch die Präposition $o$, über/ u( sich $\left._{\mathrm{DAT}}\right) \mathrm{m}$ “:
(13a) Oni obwiniali

$$
\begin{aligned}
& {\left[_ { \mathrm { VP } } \mathrm { go } \left[_{\mathrm{PP}} \mathrm{O}\right.\right.} \\
& \mathrm{ihn}_{\mathrm{AKK}}
\end{aligned}
$$$$
\text { zbrodnię]] }
$$$$
\text { sie bezichtigten }
$$$$
\text { Untat }_{\text {AKK }}
$$
(14) (On) Przypomniał sobie $\left[_{P P} \mathrm{o}\right.$

$$
\text { jego upomnieniu }
$$$$
\text { (er) erinnerte } \text { sich }_{\mathrm{DAT}} \text { an }
$$$$
\text { seine Ermahnung }
$$

Das polnische Verb szczycićsię ,sich rühmen` erfordert dagegen direkten Kasus und zwar den Instrumental:

$\begin{array}{lllll}\text { (15) Vettel szczycił } & \text { się } & \text { swoim } & \text { sukcesem } \\ \text { V. rühmte } & \text { sich }_{\text {AKK }} & \text { sein }_{\text {INST }} & \text { Erfolg }_{\text {INST }}\end{array}$

Wird das Objekt satzförmig realisiert, so wird es - genauso wie im Deutschen - mit der Subjunktion $\dot{z} e$ (dass) eingeleitet:

(16) Vettel szczycił się, że przyjechał do mety z uszkodzonymi oponami V. rühmte sich ${ }_{\mathrm{AKK}}$ dass erreichte in Ziel mit kaputten Reifen

Hauptpunkt dieses ersten Abschnittes ist die Unterscheidung von paradigmatischem („vertikalem“) und syntagmatischem („horizontalem“) Kasus. Die Bedeutung des paradigmatischen Ansatzes liegt in zweierlei: einmal ist die paradigmatische Grundlegung für Kasusvarianz die historisch alleinig einschlägige, womit systematisch Genus (im Unterschied zum modernen Sexus) zusammenhing; zum zweiten ist paradigmatische Kasusmorphologie typologisch in über 300 Sprachen verbreitet (bekannt geworden allerdings unter dem Terminus „Differential (of) object marking/DOM“, wo der spezifische paradigmatische gegenüber dem syntagmatischen Kasusbegriff nicht hergestellt wurde). 


\section{Subjektvalenz im Sonderstatus}

In dependenzgrammatischen Darstellungen wird dem Subjektargument des Verbs im Allgemeinen kein eigener Ableitunggsstatus zugemessen. Das Subjektargument hängt im Valenzbaum genau so tief wie alle anderen Objektargumente. Die Konstituentengrammatik dagegen schöpft aus den Verhaltenseigenschaften von Subjekten den Status des „(VP-)externen Arguments", wie das z.B. die linksgerichtete Valenzreihe beim Verbinfinitiv zeigt: ( ${ }^{*}$ der Sohn) der Mutter einen Kuchen backen. Das Subjektargument tritt erst bei finiter Verwendung des Verbs in den Bannkreis des lexikalischen Prädikats, es muss in einer Dependenzgraphik höher stehen als alle anderen Argumente, nämlich dort wo der Satz seine Endform über (Tempusund Modal-)Kongruenz zwischen Subjekt und Prädikat erhält.

\subsection{Subjektvalenz im typologischen Vergleich: oblique Subjekte (,quirky subjects')}

Der Sonderstatus des Subjektarguments rührt daher, dass es in der Regel nicht nur grammatische (nämlich Kongruenz-)Aufgaben übernimmt, sondern dass es darüber hinaus einen diskursbestimmten Status innehat: den des direkt Diskursbeteiligten. Das kanonisch markierte Subjekt steht in der Regel am Anfang des Satzes und ist Träger des am geringsten markierten Kasus: des Nominativs, der sich mit Ausnahme der Kopulaauxiliare (sein, werden, bleiben) nicht unter die Ergänzungskasus (in der direkten Verbalphrase/VP) mischt. Dazu gibt es allerdings eben Ausnahmen in einem bestimmten Sinne: Gefühlsprädikate (experiencer verbs) setzen in sehr vielen Sprachen statt des Subjektnominativs einen Dativ, seltener den Akkusativ.

Barðdal/Eythórsson $(2005,2009)$ haben solchen schiefen Subjektkasus anhand vor allem isländischer Beispiele syntaktischen Subjektstatus nachgewiesen. Mir/Ihn aus (17a,b,c) stünden, wenn wir Barðdal/Eythórsson folgen, als externes Argument in SpezCP, also im Vorfeld des deutschen Satzes - wo eben ein normales Subjekt hingehört.

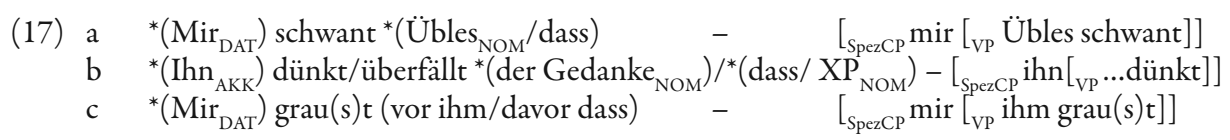

Diese satzeinleitenden Kasusobliqua sind deshalb interessant, weil sie vielfach (allerdings nicht immer im Deutschen; vgl. Barðdal/Eythórsson 2005, 2009) lexikalische Inhärenzund syntaktische Verhaltenseigenschaften wie richtige Nominativsubjekte haben. Dies gilt für eine ganze Reihe gut untersuchter Sprachen: etwa (alle der folgenden Liste aus Seržant/ Kulikov 2013).

o für spanische Antikausativkonstruktionen (d.h. „zufällige Auslöser”; vgl. Fernández Soriano /Mendikoetxea 2013, Melis/Flores 2013)

o für das oblique Pronominalobjekt der 3. Person Plural hun 'ihnen' bei belebten Referenten im Niederländischen (s. de Hoop 2013)

o für Dativsubjekte in Existentialkonstruktionen im Nordrussischen (Jung 2013)

o für Genitivsubjekte im klassischen Armenisch (Kölligan 2013) 
o für die Herausbildung nichtkannonischer Subjekte in neuindoarischen Sprachen (Hindi, Urdu) unter besonderer Sprecherperspektivik (Montaut 2013)

o für den Grammatikalisierungsweg vom Lateinischen zum modernen Italienischen von labilen Gefühlsverben wie capere „fassen, haben“ des Typs (wörtlich) „Die Angst erfasst ihn“ ebenso wie „Er fasst/ hat (die) Angst“ (Benedetti 2013; vgl. auch Abraham 2005)

o für neuere nichtkanonische Subjekte im Estnischen (Lindström 2001).

Der umgekehrte Weg von nichtkanonischen zu kanonischen Subjekten findet sich diskutiert

o fürs Altnordische bis zum modernen Skandinavischen (Faarlund 2001)

o fürs Lateinische bis zum Italienischen von me pudet 'mich beschämt“ $\mathrm{zu}$ „Ich bin beschämt/ schäme mich“ (Fedriani 2013, Abraham 2005)

o für Aspekte der Labilität von Gefühlssubjekten in daghestanischen Sprachen sowie Udi und Dargwa (Ganenkov 2013)

o für Kasuswandel zu Dativsubjekten im Baltischen und Slawischen, dies vor dem hintergrund der Noun Phrase Hierarchy bzw. Obliqueness Hierarchy (erstmals beobachtet durch Keenan/Comrie (1977) als Noun Phrase Accessibility Hierarchy; Axel Holvoet 2013)

o zum Erstspracherwerb generell oblique Subjektkonstituenten im Litauischen und Russischen (Seržant 2013).

Dies ist eine beachtliche Reihe genetisch nichtverwandter Sprachen, die alle generell unter der lexikalischen Inhärenz von Gefühlsausdrücken (experiencer verbs) oblique Subjekte entfalten. Hauptpunkt dieses Abschnittes: Die deutschen Beispiele in (17a-c) lassen sich also a priori nicht als aufs Deutsche beschränkte Unikate abtun. Hinter der Klasse der Subjektobliqua ist Grundlegendes zu sehen. Die Frage ist: Was? Zur Antwort auf diese Frage führt das folgende Kapitel.

\section{2. „Thetikvalenz"}

\subsubsection{Was ist Thetik?}

Thetische Sätze werden seit Brentano (1955-68) und Marty (1918) kategorischen Sätzen gegenübergestellt. Die linguistische Unterscheidung versucht (mit Ulrich 1985, Sasse 1987, 1995; Kuroda 1972a,b; u.a.) der philosophisch-logischen Gegenüberstellung von einfachem Urteil und doppeltem Urteil gerecht zu werden. Nach Brentano und Marty (s. auch Ulrich 1985: 57) bietet ein thetischer Satz ein einfaches Urteil/eU, ein kategorischer ein doppeltes Urteil/dU: ,... man muss die Sprache als ein Haupthindernis für die Erkenntnis ansehen, dass es auch eingliedrige Urteile, Urteile ohne logisches Subjekt und ohne logisches Prädikat gibt“ (Marty 1918:145; sekundär nach Ulrich 1985: 58). Wie sind Thetik und Kategorik linguistisch zu verorten und zu unterscheiden? Vgl. die Versuche zu einfachem Urteil bzw. Thetik in $\left(18 \mathrm{a}-\mathrm{c}^{c}\right)$.

(18) a Es regnet (Hagelschossen)

a' Solche Hageltrümmer hat es noch nie heruntergehaut

b Es laufen Kühe im Garten herum 
b، Die Kühe im Garten dürfen da nicht sein

c Es steht Wasser im Garten

c' Im Garten steht kniehoch das Schneeschmelzwasser

Die a-Beispiele gelten allgemein als thetische Sätze (einfache Urteile), die a'-Beispiele sind Muster für kategorische Sätze (doppelte Urteile). Man hat sich (vor allem nach Ulrich 1985, Kuroda 1972a,b, Fujinawa 2017, Jacobs 1991, Sasse 1995, Szabolcsi 1998) darauf geeinigt, dass die Unterscheidung nach folgenden Kriterien gelingt - die Pluswerte gelten für Kategorik (doppeltes Urteil), die Minuswerte für Thetik (einfaches Urteil):

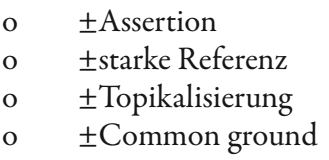

Als typische thetische Sätze gelten Existentialsätze/ sein-Prädikationen mit expletivem Es in Subjektposition (englisch There (is/are ...), französisch (il-y a...)) und damit analytische, definitorische, generische und habituelle Sätze (Ulrich 1985: 57: thetisch = ,setzend, einfach, eingliedrig (kein Subjekt, kein Prädikat) im Impersonalsatz, Existentialsatz und Satz mit Universalurteil“). Dabei gilt es zu beachten, dass Thetik keinesfalls notwendig und hinreichend intransitiver Einwertigkeit entspricht. Vgl. die eindeutigen kategorischen Sätze $\left(18 a^{c}-c^{c}\right)$ sowie (18c). Ebensowenig gälte Nullwertigkeit. Der Typus Es regnet deckt ja nicht alle Thetikfälle.

(19) a Es sind/laufen KÜHE im Garten

... thetisch

b Das Wasser steht kniehoch im Garten ...kategorisch

Nach (19a,b) scheint nun endgültig erste wichtigste einschränkende, definitorische Antworten auf die Frage, was Thetik ist, zu erlauben: Wenn $(18 \mathrm{a}, \mathrm{b}, \mathrm{c})$ und (19a) als thetisch gelten sollen, dann muss gelten:

(20) a Lexikalische Verbvalenz (Verbeinwertigkeit) alleine kann kein Kriterium für Thetik sein b eU-Status ist bei (19) gewährleistet durch Argumentintegration (Argument-Verbmerge) in vP (d.h. (Es stehen/sind) Kühe im Garten/(Es steht das) Wasser im Garten sind jeweils als einziges Argument zur Prädikation zu rechnen - damit ist syntaktische Nullwertigkeit des Satzes als thetische Satzeinwertigkeit gewährleistet. Vgl. Jacobs 1991, Sasse 1995, Szabolcsi 1998.

c Der Vergleich von (18b) und (19a) (beide thetisch) gegen (19b) (nichtthetisch) scheint dafür zu sprechen, dass das Thetiksubjekt kein Agens sein darf (also semantisches Themasubjekt haben muss). Vgl. Jacobs (1999).

d Vor allem (19a) spricht dafür, dass Betonung entscheidend für die Thetikbewertung ist (vgl. Sæbø 2007).

\subsubsection{Prosodie als Thetikkriterium - und damit Valenzkriterium}

Thetik ist als informationsstrukturelle Konfiguration beschrieben worden, die neue Objekte oder Ereignisse in den Diskurs einführt. Thetische Konstruktionen bestehen in der Regel aus 
einer morphosyntaktischen oder prosodischen Kennzeichnung in Kontrast zu einer mehr oder weniger prototypischen Subjekt-Prädikat-Phrase bzw. Topik-Kommentar-Konfiguration. Die Topik-Kommentar-Konfiguration gilt als typischste Struktur, in der ein Prädikat einem Subjekt oder Topik/Thema zugeschrieben wird, das im Adressatenbewusstsein bereits aktiviert ist. Anders gesagt: Topik-Kommentar-Konstruktionen rufen prototypische Subjekt-Prädikat-Sätze hervor wie zum Beispiel Der Hund läuft auf dem Gelände, in dem ein Subjekt der Hund heißt und ein Prädikat (die Tätigkeit des Laufens auf dem Gelände) dem Hund zugeschrieben wird. Zum Unterschied zwischen einem Topik-Kommentar- und einer Thetikkonstruktion vgl. (21a,b) (nach Garcia Macias 2015: 1-2).

(21) a. Der Papst ist GESTORBEN - The Pope DIED

b. Der PAPST ist gestorben - The POPE died (Englisch nach Schmerling 1976)

Es liegt mit (21a) und (21b) valenzsemantisch genau der gleiche Satz vor, nur die prosodische Kontur hat sich geändert. Genau diese Änderung allerdings besorgt - über ein und derselben Dependenzstruktur! - einen diskurspragmatischen Unterschied. Im Einzelnen unterscheiden sich beide Sätze in Bezug auf die Referenten, die der Sprecher beim Adressaten als aktiv voraussetzt. Satz (21a) hat ein prototypisches Intonationsmuster mit Akzent auf dem Prädikat (Akzent auf dem Subjekt wäre in diesem Fall eine zweite Option). Dies ist das Muster der prototypischen Topik-Kommentar-Konstruktionen im Englischen. In der Tat könnte dieser Satz nach dem Tod von Johannes Paul II im Jahr 2005 ausgesprochen worden sein, dessen Hinscheiden in fortgeschrittenem Alter und nach längerer Kranksein erfolgte. Der Papst und sein Gesundheitszustand waren relativ aktiv im öffentlichen Bewusstsein, und der Eintritt seines Todes waren erwartet worden. Informationsstrukturell schreibt der Satz ein Prädikat (sein Ableben) einem vorher festgelegten Themareferenten (dem Papst) zu.

Bei Satz (21b) dagegen liegt ein thetisches Intonationsmuster vor: ein deakzentuiertes Prädikat und ein akzentuiertes Subjekt. Dieses prosodische Muster gilt als Beschreibung des Stands der Dinge, der Neues und Unerwartetes vermittelt. (21b) zum Beispiel hätte 1978 zum unerwarteten Tod von Johannes Paul geäußert werden können, nur 33 Tage nachdem er erst zum Papst gewählt worden war. In diesem Fall, da das Thema im öffentlichen Bewusstsein nicht aktiv war und sein Hinscheiden nicht vorauszusehen war, wäre es seltsam gewesen die Feststellung mit der Intonation von (21a) zu machen, womit ja angedeutet war, dass der Papst und sein Leiden in allermanns Munde war. In dieser Situation war das Prosodiemuster von Beispiel (21b) das richtige. Es vermittelt, dass der Satz als Ganzes neue Information darstellt, einen unerwarteten Zustand beschreibt. Ein thetischer Satz wie (21b) ist eine Antwort auf die Frage Was ist geschehen? Was ist los?, in der Literatur auch als 'hot News' beschrieben. (22a) dagegen, die Topik-Kommentar-Konstruktion, ist eher die Antwort auf die spezifischere Frage Was ist los mit dem Papst? Wie geht es ihm? Diese Konstruktion gilt als einer der wichtigsten Subtypen der Assertion. Man könnte auch sagen: Der Thetiksatz beschreibt eine Situation oder ein Ereignis nach dessen Eigenschaft (,Papsthinscheiden'), der Kategorialsatz ordnet dem Subjektargument eine solche Ereigniseigenschaft als Träger dieser Eigenschaft zu. So gesehen kommt der Carlsonsche Begriff des essentiellen Prädikats (individual level predicate/ILP) dem des thetischen Satzes sehr nahe, der des Zustandsbegriffs 
(stage level predicate/SLP) dagegen dem des kategorischen Satzes, in dem ein Prädikatsereignis dem Subjektreferenten zugeordnet wird.

(23a,b) unterscheiden sich nach ihrer Akzentverteilungscharakteristik. Zumindest aufgrund des Unterschieds zwischen (23a) und (23b) muss gelten, dass Argument und Prädikat in c-Kommandobeziehung oder zumindest oberflächlich direkt nebeneinander (adjazent) stehen (Kriterium von Jacobs 1991).

(22) a "Kühe laufen im GARTEN (/im Garten ums HAUS) herum ... auf dem tiefsteingebetteten Kopf $=$ Defaultsatzakzent, kategorisch/ereignisprädikativ (stage level)

b KüHE laufen im Garten herum ... thetisch (akzentintegriert), ereignisprädikativ (stage level)

c Kühe laufen im Garten herum ... analytisch/generisch/definitorisch und essentiell prädikativ (individual level), daher (?) thetisch

d Es sind (die) Kühe im Garten $\quad \ldots \neq$ (22a): kategor. (thetisch)

e Da sind KÜHE im Garten ... lokativ/verortend oder 'existentiell'

Wenn aus (22a,c) ableitbar ist, dass das Prädikat betont sein muss/Akzent tragen muss, dann widerlegt dies (22b), denn (22b) ist entweder integriert- thetisch (kontextlos) oder kontrastakzentuiert (präsupponierend, andere Viecher laufen auch noch im Garten herum'). D.h. bei Integration muss das Verb keinen Extraakzent tragen (gegen Sæbø 2007: 17).

(22e) ist entweder raumzuordnend/lokativ oder, existentiell' zu lesen, offenbar ohne dass Akzent unterscheiden würde. Gibt es zu diesem semantischen Unterschied eine syntaktische Unterscheidbarkeit? Wie wäre eine solche motiviert? Heißt ,existentiell“ „etwas existiert“, oder heißt es „etwas wird präsentiert“ - letzteres etwa im Sinne von ,Figure“ und, Ground'?

$\mathrm{Zu}$ beachten ist, dass thetisch $=$ präsentativ ist, nicht lokalisierend und nicht existentiell.

(23) a Da sind KÜHE

b Es sind KÜHE

... thetisch, da rein darauf hinweisend (präsentierend)

c Es sind KÜHE im GARTEN ... nichtthetisch, da Akzent auf $K \ddot{U} H E$ auf Komplementärmenge hinweist ...thetisch, rein präsentierend

\subsubsection{Oblique Subjekte (,quirky subjects')}

Sonderfälle zur Thetik scheinen (24) zu sein, vor allem mit den Variationen $a-a$ a $-a^{\text {c }}$ und b-b"

(24) a Mir graust (vor Würmern)

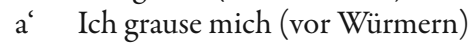

a" Es graust mich (vor Würmern)

b Mich schwindelt (beim Balancieren)

$b^{c} \quad$ Es schwindelt mich (beim Balancieren)

Barðdal/Eythórsson $(2005,2009)$ haben solchen schiefen Subjektkasus anhand vor alle, isländischer Beispiele (und unter Beachtung der Subjektkriterien, die Keenan/Comrie (1977) vorgelegt haben) syntaktischen Subjektstatus nachgewiesen. Mir/Mich aus (25a,b) stünden, 
wenn wir Barðdal/Eythórsson folgen, als externes Argument in SpezCP, also im Vorfeld des deutschen Satzes - wo eben ein normales Subjekt hingehört.

Ich meine, oblique Subjekte wie die deutschen in (25) sind in dieser spezifischen Verwendung als Gefühlsbetroffene einer semantischen Nahbeziehung ausgesetzt, die sich auch in anderen Beispielen ausdrücken lässt. Vgl. (26)-(27).

(25) a Ich breche mir ein $_{\mathrm{i}}$ Bein ${ }_{\mathrm{iAKK}}$

b Ich, breche ihm $\mathrm{i}_{\mathrm{i}}$ das Bein $_{\mathrm{i}-\mathrm{AKK}}$

c Ich, breche das Bein ${ }_{\mathrm{i} A K K}$

d Das Bein ${ }_{\text {iAKK } \rightarrow \text { NOM }}$ wird mir ${ }_{j}$ gebrochen

(26) a Es wird eifrig getanzt

b Es wird eifrig walzergetanzt

c "Der Walzer ${ }_{\text {AKK } \rightarrow \mathrm{NOM}}$ wird eifrig getanzt

brechen wie in (26) und tanzen nach (27) sind wohl transitiv-zweiwertig. Aber diese transitive Zweiwertigkeit kommt in den üblichen Probestrukturen gar nicht zum Ausdruck: es verbietet sich Passvierung (26d), und Walzer lässt sich nicht ohne weiteres als direktes Objekt verschieben. Beide Prüferscheinungen lassen sich erklären, wenn Verbinkorporation für walzertanzen und aus der Teil-von-Beziehung zwischen mir/ihm und Bein ebenso Nichtpassivierbarkeit abgelitet wird. D.h. die Valenzargumente Bein und Walzer sind der üblichen Objektbeweglichkeit entzogen: sie sind nicht frei versetzbar. (26c,d) bedeuten etwas anderes als $(26 \mathrm{a}, \mathrm{b})$, und $(27 \mathrm{c})$ ist nach Syntax und Akzentverteilung falsch. Verbvalenz wird unter bestimmten Umständen brüchig: in (26a) dadurch, dass das direkte Objekt/DO in einer Teil-von-Beziehung zum Subjekt steht, und in (27b), indem walzer verbinkorporiert als freies DO ebensowenig für Passivierung zur Verfügung steht. Wir sagen, das DO ist verbinkorporiert.

(27) a brechen:

$\begin{array}{lll}\text { a brechen: } & & {[\text { ich, mir, Bein }]} \\ \text { b tanzen: } & -[\text { walzer }] \\ \text { c grausen: } & \quad[\text { ich, vor Würmern }]\end{array}$

Valenztemplat für (26a)

Valenztemplat für (27b)

Valenztemplat für $\left(25 \mathrm{a}, \mathrm{a}^{\mathrm{c}}\right)$

Diese Valenztemplate verzeichnen keine externen Argumente/Subjekte. Es handelt sich um syntaktische Nullvalenzen, obwohl die beteiligten Prädikate transitiv-zweiwertig sind. Es passt dazu auch die grundlegende Agenseinschränkung: ein Agens kann nicht in VP auftauchen. Passivierung ist folglich unter der semantischen Übertragungseinschränkung (,Um Passvierung möglich zu machen, muss eine semantische Eigenschaft vom Agens auf das Patiens übergehen` (Abraham 2006)) unmöglich.

\subsubsection{Onoes Verben der Gefühlsempfindung als Nullvalenzen}

Wir haben gesagt, Verben mit obliquen Subjektvalenzen sind eigentlich Nullvalenzen. Wir haben solche Nullvalenzen Satzfinitmarkierungen durch japanisch -ga gleichgesetzt. Wenn nun Kuroda (1972a,b) sagt, dass thetische Sätze japanischen ga-Subjekten entsprechen, dann bleibt offen, ob auch alle ga-Subjekte auch thetische Sätze sind. Prüfen wir das: Onoe (1973) führt eine Reihe von „uneigentlichen“ $g a$-Subjekten ein, Subjekte der B-Klasse (Tanaka 2017b). Es handelt sich um (29)-(33) (nach Tanaka, pers. Hinweis): 
(28) ( ${ }^{*}$ Existentialsatz, da grundlegend $\neq$ ) Präsentationssatz:

(soko-ni) neko-ga iru.

da-LOK Katze-ga sein

Da ist „eine Katze“. Es ist da eine Katze. Es gibt da eine Katze

soko-ni wird artikuliert, wenn ein bestimmter Ort profiliert ist. Sprechakt der Existenzfeststellung ist grundlegend etwas anderes als der Sprechakt der Präsentation (des neu Aufdentischlegens; neu im CG Ablegens; eine neue $\mathrm{QuD}$ setzend).

(29) Objekt des Gefühls:

Furusato-ga natsukasii.

Heimat-ga Sehnsucht-erwecken

„Ich sehne mich nach meiner Heimat“ (meine Heimat im Jap. in Subjektfunktion: , meine Heimat weckt meine Sehnsucht'/'macht mich sehnsüchtig')

(30) Objekt des eigenen Vermögens/Könnens:

$\begin{array}{lll}\text { (Watashi-wa) } & \text { chugokugo-ga } & \text { dekiru. } \\ \text { Ich-TOP } & \text { Chinesisch-NOM } & \text { können }\end{array}$

Ich kann „Chinesisch“/ Ich bin „des Chinesischen“ kundig, wobei im Japanischen „Chinesisch“ als Subjekt auftritt

(31) Entität des „spontanen Gefühlsaufkommens“ (Onoe 1973/Tanaka 2017b; ’= „des sponta nen Empfindens"):

Furusato-ga natsukasii.

Heimat-ga Sehnsucht-erwecken

Ich erinnere mich an „meine Heimat", wobei im Japanischen „meine Heimat" als Subjekt kodiert wird

(32) Sitz der Empfindung:

Atama-ga itai.

Kopf-ga tut-weh

„Der Kopf“ tut mir weh. (vgl. (27) und (34a): *(sich) ein Bein brechen)

Wir plädieren, (29)-(33) (mit Leiss 2000) ähnlich wie (34a), nicht jedoch (34b) zu sehen, also als Teil-von-Semantiken, die wiederum VP-inkorporierte Syntaxstrukturen haben und da als Nullvalenzen zu identifizieren sind.

(33) a Er bricht sich ein Bein? ( ${ }^{*}$ Er bricht ein Bein)

b *Ein Bein wird $\left({ }^{*}\right.$ sich $)$ durch ihn $/{ }^{*}$ sich gebrochen.

D.h. sich ein Bein brechen $\neq$ ein Bein brechen, aber = break a leg. Es lässt sich das Subjektargument nicht vom Prädikat trennen, syntaktisch also wie (28)-(32). (34a-f) verzeichnen weitere solche Pseudotransitivsätze und Pseudosubjekte.

(34) a $\left[{ }_{\mathrm{CP}}\right.$ Es brach $\left[_{\mathrm{VP}}\right.$ er sich ein Bein $\left.]\right] \neq\left[_{\mathrm{CP}} \operatorname{Er}\right.$ (zer)brach $\left[{ }_{\mathrm{VP}}\right.$ ein Bein $\left.]\right]$

b $\quad\left[{ }_{\mathrm{CP}}\right.$ Es sehne $\left[{ }_{\mathrm{VP}}\right.$ ich mich nach meiner Heimat $\left.]\right]$

c $\quad\left[{ }_{\mathrm{CP}}\right.$ Es kann $\left[{ }_{\mathrm{VP}}\right.$ ich Chinesisch] $]$ bzw. $\left[_{\mathrm{CP}}\right.$ Es bin [ ${ }_{\mathrm{VP}}$ ich des Chinesischen kundig] $]$

d $\quad\left[{ }_{C P}\right.$ Es tut $\left[{ }_{\mathrm{Vp}}\right.$ mir der Kopf weh] $]$

e Mir ist kalt - Ich habe es kalt

f Ich habe Hunger - Ich bin hungrig (- russisch/lateinisch, (Bei) Mir ist Hunger/Mihi fames est') 
(34a-f) signalisiert Teil-von-Subjekte, bei denen mit Blick auf Transitivität und Passivierung keine Eigenschaftsübertragung vom Subjekt auf das Objekt stattfindet (vgl. Abraham 2006):

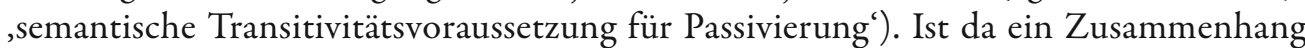
zu Onoes (1973) typischen ga-Verben zu sehen: mit Blick auf die Unmöglichkeit zu passivieren und letztens auf Thetik?

Alle von Onoes (1973) B-Klassensubjekte haben klare Affinität zu -ga, sie lassen sich mit - wa nur mit Mühe kombinieren. D.h. auch diese Subjekte sind grundsätzlich „thetische Subjekte" (was bei Kuroda völlig unberücksichtigt bleibt), d.h. es sind VP-inkorporierte Subjekte, sie treten entsprechend strukturell als komplette Proposition, d.h. ohne syntaktisch (wohl aber semantisch) externes Argument auf. Andere „normale“ Subjekte können sowohl in VP als auch außerhalb stehen, wobei -wa grundsätzlich die CP-Subjekte markiert (Kategorik), -ga dagegen VP-Subjekte (Thetik). VP-Subjekte lassen sich allerdings über entsprechenden Kontext auch als externe Subjekte, also als Subjekte kategorischer Sätze interpretieren (wobei laut Tanaka (pers. Mitteilung) auf -ga leichter Akzent fällt, also intonatorisch markiert ist).

\subsubsection{Thetik, Subjektinkorporation und die japanische ga-Verbklasse}

Was kann zur Eigenständigkeit von Onoes ga-Verbalität semantisch-pragmatisch (eventuell ähnlich Truckenbrodts (2006) Ansatz argumentierend) beitragen? Was verbindet Kurodas ga-Syntax (Thetik, Subjektinkorporation) mit Onoes spezieller ga-Verbklasse in (29)-(32)? Wir sagen: Subjekte der speziellen ga-Prädikatsklasse zeichnen sich aus durch:

(35) a Nichtbesetzung des Vorfelds bzw. von Spez-CP und damit Extradiskursstatus

b CG-losigkeit und trotzdem Vollpropositionsstatus

c = autonomer (= von keinem anderen Operator abhängigen) Semantik

$\mathrm{d}=$ strukturelle Superintransitivität

e = „Urpropositionalität“; vgl. Abraham 2007: „Es gibt keine Protointransitivität“

$\mathrm{f} \approx$ markierte (die Proposition insgesamt fokussierende) Akzentsetzung/Intonatorik

$\mathrm{g} \approx$ Exklamativität (im D auch außerhalb des syntaktischen Rootkanons; keine QuDQuaestio)

Mit Onoe (1973; vgl. auch Tanaka 2017a,b) klammern sich Verben der Gefühlsempfindung insofern aus der allgemeinen Klasse der japanischen Verben aus, als sie nur mit dem Verbsuffix - ga finit gemacht werden können. Finitheit ist üblicherweise an die Suffixe -wa und -ga gebunden. -wa geht mit diskursgrammatischer Verwendung einher, -ga dagegen bleibt eine rein grammatische Einzelsatzcharakterisierung. Diese Verteilung steht zu unseren deutschen Beispielen insofern, als VP-externe Argumente diskursgebunden sind, VP-interne dagegen nicht. Gefühlsverben wie sich etwas brechen und (sich) grausen sind im Sinne Onoes ga-Verben, also reine (argument-/subjektinkorporierende) VP-Template. Sie entziehen sich in diesem Sinne der reinen Beurteilung unter lexikalischem, dependenztheoretischem Gesichtspunkt. 


\subsubsection{Unakkusativa mit VP-internem Subjekt}

Beziehen wir allerdings unakkusativische Verben (= perfektive Intransitiva) mit in die Templatüberlegungen mit ein, ergibt sich die Schwierigkeit, wie sich Unakkusativsubjekte und VP-inkorpororierte Subjekte unterscheiden. Unakkusativsubjekte haben ja VP-interne Eigenschaften.

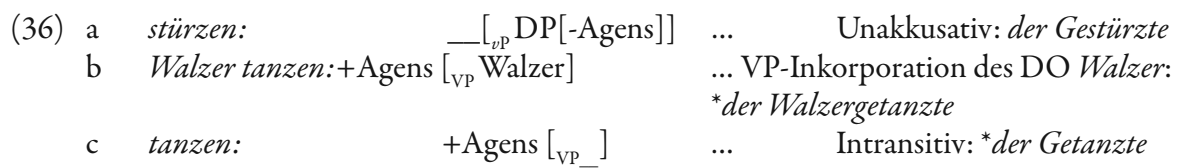

Wir haben die VP-Schale um einen Ring erweitert: $v$ P um VP herum wie in (36a) gegen (36 b,c). Das Unakkusativsubjekt steht strukturell höher als das VP-inkorporierte Subjekt. Das ist eine kanonische Annahme in der modernen Syntax (Abraham 2013: Kap. 9). Wird Fokus mitangezeigt, ergeben sich diese Darstellungen. $\left[\mathrm{Es}_{\mathrm{EXPL}}=\right.$ expletives (lexikalisch leeres) Subjektargument]

(37) a $\left[{ }_{\mathrm{CP}} \mathrm{Es}_{\mathrm{EXPL}}\left[_{\mathrm{VP}} \text { Argument }_{\mathrm{F}} \mathrm{V}\right]\right]_{\mathrm{F}} \ldots$ für thetisches, VP-inkorporiertes Externargument

$$
\begin{gathered}
\mathrm{b} \quad\left[_{\mathrm{CP}} \mathrm{Es}_{\mathrm{EXPL}}\left[{ }_{\nu \mathrm{P}} \text { Argument }_{\mathrm{F}} \mathrm{V}\right]\right]_{\mathrm{F}} \ldots \text { für unakkusativisch kategorisches } v \text { P-internes } \\
\text { Externargument (Subjektargument/-valenz) }
\end{gathered}
$$

\subsubsection{Deklaratives V1 als Thetik: Bühnenanweisung}

Zum Sprechakt „Bühnenanweisung“ als deklarativem V1:

(38) a AUF-/EIN-/ABtritt Macbeth.

b Enters MacBeth

c Macbeth(Makubesu)-ga toujou-suru. (Tanaka, pers. Mittlg.)

Macbeth-ga auftreten.INF

Hier nimmt das Verb die japanische Grundform (infinite Form) an, was allerdings sehr selten ist (eventuell außer mit der ersten Person, wobei die konkrete Absicht des Sprechers zum Ausdruck gebracht wird). D.h. die Bühnenanweisung wird quasi „infinitivisch“ (wie die deutsche imperativische Ansageform) gesetzt. Wenn es dagegen um eine Sachverhaltsschilderung mit der dritten Person geht, weicht man von der Infinitsetzung des Prädikats ab und fügt einen Modalitäts- bzw. Evidentialitätsausdruck hin. Wie immer auch, die ga-Markierung entspricht dem Thetikverständnis - und damit Nullvalenzverständnis - dieses V1-Sprechakts. Solche deklarative V1-Strukturen lassen sich als solitäre VPs, somit als Nullvalenzen verstehen.

V1-Deklarativformen sind in den nordischen Sagas immer dann im Einsatz, wo es um narratives Verhalten geht, um die Spannung beim Leser zu erhöhen: etwa bei Angriffsszenen bei Kampfschilderungen (Leiss 2007, Abraham 2011). Dies ist wohl parallel zu sehen zu Witzanfängen: 
(39) Sagt der Hase zum Igel $_{F} /$ Betritt ein Matrose $_{\mathrm{F}}$ eine Hafenkaschemme . $^{\text {. }}$

Ganz deutlich seht das finite Prädikat hier in Comp, der ersten Verbklammer, und lässt alles andere an Satzgliedern in VP. Wollen wir dem Umstand Rechnung tragen, dass hiermit spezielle Sprechakte charakterisiert sind, müssen wir von Nullvalenz unter dieser Sprechaktverwendung des Prädikatsverbs sprechen. Dass es sich dabei um Nullvalenz unter VP-Inkorporation aller Argumente handelt, verleiht dieser Form einen eigenen Status: den einer Ereignisganzheit, soz. dem ungeteiltetsten Anfang eines Gedankens, in dem sich das gesamte Bild des Gedankens in den Vordergrund hebt, wo sich die kompositionelle Syntax aus den Satzgliedern noch nicht entwickelt hat, wo aber gleichwohl die Einzelstücke zum syntaktisch-kompositorischen Prozess bereits zur Verfügung stehen. Projiziert auf die minimalistische Idee des Mergevorgangs entspricht dies dem bereits lexikalisch angereicherten Syntaxskelett, das aber die einzelnen Kopf- und Abhängigkeitsstrukturen noch nicht abgerufen hat.

\section{Schlussfolgerungen: die volatile Subjektvalenz}

Wir haben eingangs die folgenden Fragen zu dieser Untersuchung gestellt:

(i) Verstehen wir Valenz, also als paradigmatische oder als syntagmatische Erscheinung?

(ii) Beziehen wir zur Valenzbegrifflichkeit thetischen Kategorienstatus mit ein?

(iii) Was für eine Rolle spielt Valenz in Sprachen, in denen diskursgrammatische Argumentthematisierung deren Grammatik beherrscht?

Wir schließen die Antwort auf (iii) schnell ab. Sie betrifft ausschließlich das Subjektargument sogenannter Topiksprachen, welches unter Themaellipse soz. bloß oberflächlich aus dem Valenzraster verschwindet, jedoch zu jeder Zeit rekonstruierbar bleibt. Zu den anderen beiden Fragen, (i) und (ii), haben wir folgende Resultate gefunden, die ich hier in neuem Format vorstelle. Wir unterscheiden diese vier Valenztypen. Ich stelle das Prädikat V natürlich an das Satzstrukturende, von wo aus die Prädikatsvalenz bis zum Vorfeld hin aufgebaut wird. [SpezCP = Subjekt- bzw. Vorfeldposition; PART = Partikel, PRÄF = Präfix, A = Adjektiv $]$

(40) a $\left[_{\text {SpezCP }} \mathrm{XP}\left[{ }_{v \mathrm{P}}-\left[{ }_{\mathrm{VP}} \mathrm{YP} Z \mathrm{ZP}\right]\right] \mathrm{V}\right]$

...syntagmatische Valenz - externes Subjektargument, Objekte YP-ZP serialisiert (unter verschiedenen Satzgliedern) valenzidentifiziert

b $\quad\left[_{\text {SpezCP }} \mathrm{XP}\left[{ }_{v \mathrm{P}}-\left[{ }_{\mathrm{VP}} \mathrm{Y} / \mathrm{ZP}\right]\right] \mathrm{V}\right]$

...paradigmatische Valenz - externes Subjektargument, Objekte YP-ZP unter einem einzigen Satzglied valenzidentifiziert c $\quad\left[{ }_{S \mathrm{pez} C \mathrm{P}}-\left[_{v \mathrm{P}} \mathrm{XP}\left[{ }_{\mathrm{VP}} \mathrm{YP} Z \mathrm{PP}\right]\right] \mathrm{V}\right]$

...Unakkusativvalenz - $(v \mathrm{P}$-)internes Subjektargument

d $\quad\left[{ }_{\text {SpezCP }}-\left[{ }_{\mathrm{vP}}-\left[{ }_{\mathrm{VP}} \mathrm{XP}\right.\right.\right.$ YP ZP $\left.\left.]\right] \mathrm{V}\right]$

...Thetikvalenz - VP-inkorporiertes Subjektargument

e $\quad\left[_{\text {SpezCP }} \mathrm{XP}\left[_{\mathrm{VP}}\left[\left[_{\mathrm{VP}}[\right.\right.\right.\right.$ YP Part-/A- $\left.\left.]\right]-\mathrm{V}\right]$

...kausative Partikelverbvalenz - transitiv: $E I N_{P A R T} s c h l a ̈ f e r n$,

*(sich) SCHÖN schlafen, $^{*}$ (etwas) der ${ }_{P R A F}$ rennen 
f $\quad\left[_{\text {SpezCP }} X P\left[_{\mathrm{vP}}\left[\left[_{\mathrm{VP}}[\right.\right.\right.\right.$ Part-/A- $\left.\left.]\right]-\mathrm{V}\right]$

...antikausative Partikelverbvalenz - intransitiv: $E I N_{\text {PART }}{ }^{-/ S C H O ̈ N_{A}}$ schlafen, *(sich) der ${ }_{P R A F}$ rennen

(40e,f) zeichnen die üblichen Kausativ- und Antikausativvalenzen nach. $\mathrm{Zu}$ (40d) nehme ich an, dass die lexikalisch-dependentiellen Valenzbeziehungen bei Thetikvalenz vorhanden sind. Dies muss schon deshalb gelten, weil sie auch für Unakkusativverben gelten: Das Unakkusativsubjekt hat Eigenschaften des direkten Objekts; ein Subjekt dieser Eigenschaft ist selegiert durch perfektive Intransitiva. Die Oberflächenposition des Unakkusativsubjekts wird durch die Syntax festgelegt: Es gibt keine deutschen Sätze ohne Vorfeldfüllung. Dies ist aber eine Lizensierungsbedingung, die von valenz- bzw. dependenztheoretischen Kriterien völlig unberührt bleibt. Es lässt sich also folgern, dass auch unter thetischer Valenzstrukturierung, also bei VP-Inkorporation aller Valenzargumente die dependentielle Strukturierung erhalten ist - dies vor allem deshalb, weil propositionelle Semantik zur Interpretation führt. Unter VP-Inkorporation liegt uneingeschränkte semantische Interpretierbarkeit vor, allerdings als Ereigniseigenschaft wie bei essentiellen Prädikaten (Individual Level Predicates/ ILP). Dem ist (40c) mit dem Subjekt in einer Objektvalenzeigenschaft (in der $v$ P-Schale) nahe. Alle anderen Valenzvariationen in (40) kennzeichnen die satzglieddekomponierte Valenzstruktur.

Hauptpunkt allerdings ist wiederum die formale Unterscheidung des thetischen Satzes als „VP-Proposition“, als totale Satzgliedinkorporation in VP und damit als Ereigniseigenschaft. Ich deute dies als Gesamtbild des Ereignisses - sozusagen als Kategorie der Ursyntax. Dazu stimmen die V1-Satztypen der Bühnenanweisung („,mit dem Auftritt von X beginnt eine neue Situation“), der einhaltgebietenden Kampfschilderung vor allem in den frühnordischen Sagas (Leiss 1992, Abraham 2011), Witzeinleitungen, Gefühlsausdrücke (Mir tut der Kopf weh!), Teil-von-Semantiken (Er ${ }_{i}$ brach sich ${ }_{i}$ das ${ }_{i}$ Bein) - Thetik eben. Dafür, meine ich, steht auch (gegen Kuroda 1972a,b) japanisch ga, während wa ebenso wie kategorische Sätze Prädikate bestimmten Argumenten (Komplementen, Valenzträgern) zuordnen (wo es keine Referenzidentität ${ }_{i}$ zwischen den Satzgliedern $_{i}$ geben kann). Wie alle Satzglieder in VP fallen auch Figur und Grund in einer Grundballung zusammen.

Es darf nicht darüber hinweggesehen werden: Wir haben mit der Identifikation von Valenzdiversifikationen wie in (40) nicht nur Unkanonisches zur Valenztheorie zu sagen. Es ging auch darum, die älteren Begriffe Thetik und Kategorik über ein neues Valenzverständnis genauer linguistisch zu erfassen. Und schließlich ging es darum, Kurodas (1972a.b) Gleichsetzung von , thetisch = japanisch ga/Nominativmorphologie und kategorisch $=$ japanisch $w a /$ diskursthematisch 'durch eine ganz andere Einordnung zu ersetzen. wa kennzeichnet ebenso wie $g a$ eine narrative Kategorie: wa ist in den narrativen Fluss einordnend, $g a$ dagegen narrativ aufhaltend, unterbrechend und damit isolierend und Aufmerksamkeit erregend. Man wird sehen, wie die Japanologie (etwa im Anschluss an Onoe 1973) auf diese These reagiert. 


\section{Literatur}

Abraham, Werner (1997): The interdependence of case, aspect, and referentiality in the history of German: the case of the genitive. In: Ans van Kemenade \& Nigel Vincent (eds.): Parameters of morphosyntactic change, 29-61. Cambridge: Cambridge University Press.

Abraham, Werner (2006a.): Introduction: Passivization and typology. Form vs. function - a confined survey into the research status quo. In: W. Abraham \& L. Leisio (eds.): Passivization and typology. Form and function, 1-28. [Typological Studies in Languages 68]. Amsterdam: John Benjamins.

Abraham, Werner (2006b.): The compositional nature of the analytic passive: Syntactic vs. event semantic triggers. "Argument Hypothesis" vs. "Aspect Hypothesis". In: Werner Abraham \& Larisa Leisiö (eds.): Passivization and typology. Form and function, 462-501. [Typological Studies in Languages 68]. Amsterdam-Philadelphia: John Benjamins.

Abraham, Werner (2007): Types of transitivity, intransitive objects, and untransitivity - and the logic of their structural designs: Ways to keep apart derivation in syntax and in the lexicon. In: Patrick Brandt \& Marco García García (eds.): Transitivity. Form, meaning, acquisition, and processing, 15-68. [Linguistik Aktuell/Linguistics Today 151]. Amsterdam: John Benjamins.

Abraham, Werner (2011): Linke Satzperipherie und versteckte Definitheitskategorie: V1-Deklarative, Wortartunabhängigkeit und Narrativität. (Althoch)Deutsch - Altisländisch im Vergleich. In: M.L. Kotin \& E.G. Kotorova (Hg.) unter Mitarbeit von M. Durrell. Geschichte und Typologie der Sprachsysteme, 49-68. Heidelberg: C. Winter.

Abraham, Werner (2013): Deutsche Syntax im Sprachenvergleich. 3. Auflage. Tübingen: Narr/ Stauffenburg.

Abraham, Werner (2016): Valency frames at large. 'Vertical' vs. 'Horizontal' case government. Talk and presentation ÖLT 2016 Graz.

Abraham, Werner \& Elisabeth Leiss (2012): The case differential: Syntagmatic versus paradig-matic case - its status in synchrony and diachrony. Transactions of the Philological Society 110:316-341. London: Wiley.

Abraham, Werner \& Elisabeth Leiss (2017): Multiple case binding - The principled underspecification of case exponency. In: Lars Hellan, Andrej Malchukov \& Michela Cennamo (eds.): Contrastive studies in verbal valency, 27-82. [Linguistik Aktuell 237]. Amsterdam: John Benjamins.

Aissen, Judith (2000): Differential Object Marking: Iconicity vs. economy. Ms. UCSC MAY, 2000.

Barðdal, J. \& Th. Eythórsson (2005): Oblique subjects: A common Germanic inheritance. Language 81(4): 824-81.

Barðdal, Johánna \& Thorhálldor Eythórsson (2009): The origin of the oblique subject construction: An Indo-European comparison. In: Grammatical Change in Indo-European Languages, ed. by Bubenik, Vit, John Hewson \& Sarah Rose. Amsterdam: John Benjamins. 179-193.

Bossong, Georg (1991): Differential object marking in Romance and beyond. In: D. Kibbee \& D. Wanne (eds.): New Analyses in Romance Linguistics, 43-70. Amsterdam: Benjamins.

Brentano, Franz (1955-68): Psychologie vom empirischen Standpunkt, 3 Bde. Hamburg: Meiner.

Carlson, Gregory N. (1995): Truth Conditions of Generic Sentences: Two Contrasting Views. In: Gregory N. Carlson \& Francis Jeffry Pelletier (eds.): The Generic Book. Chicago: Chicago University Press.

Engel, Ulrich (1996): Deutsche Grammatik. 3., korrigierte Auflage. Heidelberg: Julius Groos.

Engel, Ulrich (2004): Deutsche Grammatik. München: Iudicium.

Engel, Ulrich \& H. Schumacher (1978): Kleines Valenzlexikon deutscher Verben. 2. Auflage. Tübingen. 
Faarlund, Jan Terje (2001): From Ancient Germanic to modern Germanic languages. In: Haspelmath, M., et al. (eds.): Language Typology and Language Universals. Sprachtypologie und sprachliche Universalien. Ein internationales Handbuch, 1706-1719. Berlin: de Gruyter [HSK; 20.2].

Fujinawa, Yasuhiro (2017): Licht und Schatten der kategorischen/thetischen Aussage: Kopula und Lokalisierungsverben im deutsch-japanischen Vergleich. In: E. Leiss, S. Tanaka, W. Abraham \& Y. Fujinawa (Hg.): Thetik-Kategorik und OV-Varianz im Deutschen und Japanischen. [Sonderheft 24 der Linguistischen Berichte]. Hamburg: Buske.

Jacobs, Joachim (1991): Integration. In: M. Reis (ed.): Wortstellung und Informationsstruktur, 64-116. Tübingen: Niemeyer.

Jacobs, Joachim (2009): Valenzbindung oder Konstruktionsbindung? Eine Grundfrage der Grammatiktheorie. ZGL 33. DOI 10.1515/ZGL.2009.033

Karlsson, Fred (1982): Finnische Grammatik. Hamburg: Buske.

Keenan, Edward L. \& Bernard Comrie (1977): Noun phrase accessibility and Universal Grammar. Linguistic Inquiry 8 (1): 63-91.

Koenig, Jean-Pierre und Karin Michelson (2015): Invariance in argument realization: the case of Iroquoian. Language 91/1: 1-47.

Kuroda, S.Y. (1972a.): Anton Marty and the Transformational Theory of Grammar. Foundations of Language 9.1-37.

Kuroda, S.Y.(1972b.): The categorical and the thetic judgment: Evidence from Japanese syntax. Foundations of Language 9.9. 153-85.

Kuroda, S.Y. (2005): Focusing on the matter of Topic: A study of $W a$ and $G a$ in Japanese. Journal of East Asian Linguistics 14. 1-58.

Leiss, Elisabeth (1992): Die Verbalkategorien des Deutschen. Ein Beitrag zur Theorie der sprachlichen Kategorisierung. Berlin: Walter de Gruyter [Studia linguistica Germanica; 31].

Leiss, Elisabeth (2000): Artikel und Aspekt. Die grammatischen Muster von Definitheit. Berlin / New York: Walter de Gruyter [Studia linguistica Germanica; 55].

Leiss, Elisabeth (2002a.): Die Rolle der Kategorie des Aspekts im Sprachwandel des Deutschen: ein Überblick. In: Japanische Gesellschaft für Germanistik (ed.): Grammatische Kategorien aus sprachhistorischer und typologischer Perspektive: Akten des 29. Linguistenseminars (Kyoto, 2011). München: Iudicium. 9-25.

Leiss, Elisabeth (2002b.): Der Verlust der aspektuellen Verbpaare und seine Folgen im Bereich der Verbalkategorien des Deutschen. In: Japanische Gesellschaft für Germanistik (ed.): Grammatische Kategorien aus sprachhistorischer und typologischer Perspektive: Akten des 29. Linguistenseminars (Kyoto, 2011). München: Iudicium. 26-41.

Leiss, Elisabeth (2007): Covert patterns of definiteness/indefiniteness and aspectuality in Old Icelandic, Gothic, and Old High German. In: Abraham, Werner / Stark, Elisabeth / Leiss, Elisabeth (eds.): Nominal Determination. Typology, context constraints, and historical emergence. Amsterdam / Philadelphia: John Benjamins [Studies in Language Companion Series; 89]. 73-102.

Leiss, Elisabeth; Shin Tanaka, Werner Abraham, Yosihoru Fuhinawa (Hg.) (2017): Grammatische Funktionen aus Sicht der japanischen und deutschen Germanistik. [Linguistische Berichte Sonderheft 24]. Hamburg: Buske.

Marty, Anton (1918): Spezielles über den Ausdruck der Urteile und die diesbezüglichen inneren Sprachformen. In: Josef Eisenmeier, Alfred Kastil und Oskar Kraus (Hg.): Anton Marty: Gesammelte Schriften, 2. Bd., 1. Abt., Halle a.S.: Niemeyer, 223-301. 
McFadden, Thomas (2004): The position of morphological case in the derivation: A study on the syntax-morphology interface. Ph.D. dissertation University of Pennsylvania.

Onoe, Keisuke (1973): Sentence kernel and sentence frame with special reference to the usage of the particles $w a$ and ga. 言 語研 究 [Gengo Kenkyu] 63: 1-26.

Piskorz, Jadwiga Klara (2012): Die Grammatikalisierung eines neuen Perfekts im Polnischen. Ein Beitrag zur Entwicklungslogik des Perfekts. München etc.: Otto Sagner.

Piskorz, Kinga Klaudia (2017): Die Herausbildung eines bestimmten Artikels im Polnischen. Der grammatische Status des Demonstrativpronomens ten und seine Rolle im Definitheitssystem des Polnischen. Dissertation Ludwig-Maximilians-Universitat München.

Sæbø, Kjell Johan (2007): Focus interpretation in thetic statements: Alternative semantics and Optimality Theory pragmatics. Journal of Logic, Language and Information 16: 15-33.

Sasse, Hans-Jürgen (1987): The thetic/categorical distinction revisited. In: Linguistics, 25. 511-580.

Sasse, Hans-Jürgen (1995): Theticity and VS-order: a case study. Sprachtypologie und Universalienforschung 48: 3-31.

Seržant, Ilja A. \& Leonid Kulikov (eds.) (2013): The diachronic typology of non-canonical subjects. [Studies in Language Companion Series 130]. Amsterdam: Benjamins.

Streitberg, Wilhelm (1971): Die gotische Bibel. Teil 1: Der gotische Text und seine griechische Vorlage. Heidelberg: Universitätsverlag Winter. [Germanistische Bibliothek, Band: 3].

Szabolcsi, Anna (1986): From the definiteness effect to lexical integrity. In: W. Abraham \& S. de Mey (eds.): Topic, focus and configurationality, 321-348. Amsterdam: John Benjamins.

Tanaka, Shin (2017a.): Suche nach latenter Invarianz bei genetisch fremden Sprachen am Beispiel Deutsch-Japanisch. In: E. Leiss, S. Tanaka, W. Abraham \& Y. Fujinawa (Hg.): Thetik-Kategorik und OV-Varianz im Deutschen und Japanischen. [Sonderheft 24 der Linguistischen Berichte]. Hamburg: Buske.

Tanaka, Shin (2017b.): V2-Puzzle 3: Thetik-Kategorik als universale konzeptuelle Struktur. Präsentation Linguistenseminar 2017 der JGG, Kyoto 5.9.2017.

Tesnière, Lucien (1959): Élements de syntaxe structurale. Paris: Klincksieck.

Tesnière, Lucien (1980): Grundzüge der strukturalen Syntax. Hrsg. und übers. von Ulrich Engel. Stuttgart: Klett.

Truckenbrodt, Hubert (2006): On the semantic motivation of syntactic verb movement to C in German. Theoretical Linguistics 32: 257-306.

Ulrich, Miorita (1985): Thetisch und Kategorisch: Funktionen der Anordnung von Satzkonstituenten am Beispiel des Rumänischen. Tübingen: Gunter Narr Verlag. 